\title{
The H.E.S.S. II GRB observation scheme
}

Cite as: AIP Conference Proceedings 1792, 050034 (2017); https://doi.org/10.1063/1.4968980 Published Online: 04 January 2017

R. D. Parsons, F. Schüssler, T. Garrigoux, A. Balzer, M. Füssling, C. Hoischen, M. Holler, A. Mitchell, G. Pühlhofer, G. Rowell, S. Wagner, E. Bissaldi, P. O'Brien, P. H. T. Tam, and the H. E. S. S. Collaboration

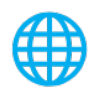

\section{ARTICLES YOU MAY BE INTERESTED IN}

Target of opportunity observations of blazars with H.E.S.S.

AIP Conference Proceedings 1792, 050029 (2017); https://doi.org/10.1063/1.4968975

The HAWC GRB programme

AIP Conference Proceedings 1792, 050033 (2017); https://doi.org/10.1063/1.4968979

The exceptional flare of Mrk 501 in 2014 combined observations with H.E.S.S. and FACT AIP Conference Proceedings 1792, 050019 (2017); https://doi.org/10.1063/1.4968965

\section{Conference Proceedings}

\section{Get $30 \%$ off all print proceedings!}




\title{
The H.E.S.S. II GRB Observation Scheme
}

\author{
R.D. Parsons ${ }^{1, a)}$, F. Schüssler ${ }^{2}$, T. Garrigoux ${ }^{3}$, A. Balzer ${ }^{4}$, M. Füssling ${ }^{5}$, C. \\ Hoischen $^{6}$, M. Holler ${ }^{7}$, A. Mitchell ${ }^{1}$, G. Pühlhofer ${ }^{8}$, G. Rowell ${ }^{9}$, S. Wagner ${ }^{10}$, E. \\ Bissaldi $^{11}$, P. O'Brien ${ }^{12}$, P.H.T. Tam ${ }^{13}$ and the H.E.S.S. Collaboration \\ ${ }^{1}$ Max-Planck-Institut für Kernphysik, Heidelberg, Germany \\ ${ }^{2}$ DSM/Irfu, CEA Saclay, F-91191 Gif-Sur-Yvette Cedex, France \\ ${ }^{3}$ Centre for Space Research, North-West University, Potchefstroom 2520, South Africa \\ ${ }^{4}$ GRAPPA, Anton Pannekoek Institute for Astronomy, University of Amsterdam, Amsterdam, The Netherlands \\ ${ }^{5}$ DESY, Zeuthen, Germany \\ ${ }^{6}$ Institut für Physik und Astronomie, Universität Potsdam, Potsdam, German \\ ${ }^{7}$ Laboratoire Leprince-Ringuet, Palaiseau, Ecole Polytechnique, CNRS/IN2P3, France \\ ${ }^{8}$ Institut für Astronomie und Astrophysik, Universität Tübingen, Tübingen, Germany \\ ${ }^{9}$ School of Chemistry $\mathcal{F}$ Physics, University of Adelaide, Adelaide, Australia \\ ${ }^{10}$ Landessternwarte, Universität Heidelberg, Königstuhl, Heidelberg, Germany \\ ${ }^{11}$ INFN - Sez. di Bari, Via E. Orabona 4, Bari, Italy \\ ${ }^{12}$ Department of Physics and Astronomy, University of Leicester, Leicester, United Kingdom \\ ${ }^{13}$ Institute of Astronomy and Space Science, Sun Yat-Sen University, Guangzhou, China \\ ${ }^{a}$ Corresponding author: daniel.parsons@ @mpi-hd.mpg.de
}

\begin{abstract}
Gamma-ray bursts (GRBs) are some of the Universe's most enigmatic and exotic events. However, at energies above 10 $\mathrm{GeV}$ their behaviour remains largely unknown. Although space based telescopes such as the Fermi-LAT have been able to detect GRBs in this energy range, their photon statistics are limited by the small detector size. Such limitations are not present in ground based gamma-ray telescopes such as the H.E.S.S. experiment, which has now entered its second phase with the addition of a large $600 \mathrm{~m} 2$ telescope to the centre of the array. Such a large telescope allows H.E.S.S. to access the sub 100-GeV energy range while still maintaining a large effective collection area, helping to potentially probe the short timescale emission of these events.

We present a description of the H.E.S.S. GRB observation programme, summarising the performance of the rapid GRB repointing system and the conditions under which GRB observations are initiated. Additionally we will report on the GRB followups made during the 2014-15 observation campaigns.
\end{abstract}

\section{Introduction}

GRBs are some of the most energetic and distant astronomical objects yet discovered. Although their source it is not yet certain, their most likely progenitors are collapsing massive stars (long GRBs) and merger events (short GRBs). So far they have been detected in a number of different energy ranges of the electromagnetic spectrum, even up to $\mathrm{GeV}$ energies (e.g. [1]) which makes them a prime candidate for observation by very high energy telescopes such as H.E.S.S. (e.g. $[2,3,4,5])$.

Due to their highly energetic nature, GRBs may also be a source of ultra-high-energy cosmic rays (UHECRs; [6]), as well as gravitational waves and neutrinos. By observing and attempting to understand the nature of GRBs it is therefore possible to attempt to answer many important scientific questions. Such as, understanding the physics of the jet likely launched from the GRB, the search for UHECR acceleration from the GRB jets, the attenuation of the highest energy gamma-rays by the extra-galactic background radiation fields and tests for Lorentz invariance violation (e.g. [7]) in these energetic distant sources.

The H.E.S.S. gamma-ray telescope array has recently entered its second phase by the addition of a fifth, almost $600 \mathrm{~m}^{2}$ telescope to the centre of the array (CT 5), greatly improves the energy threshold of the instrument $(\sim 50$ 


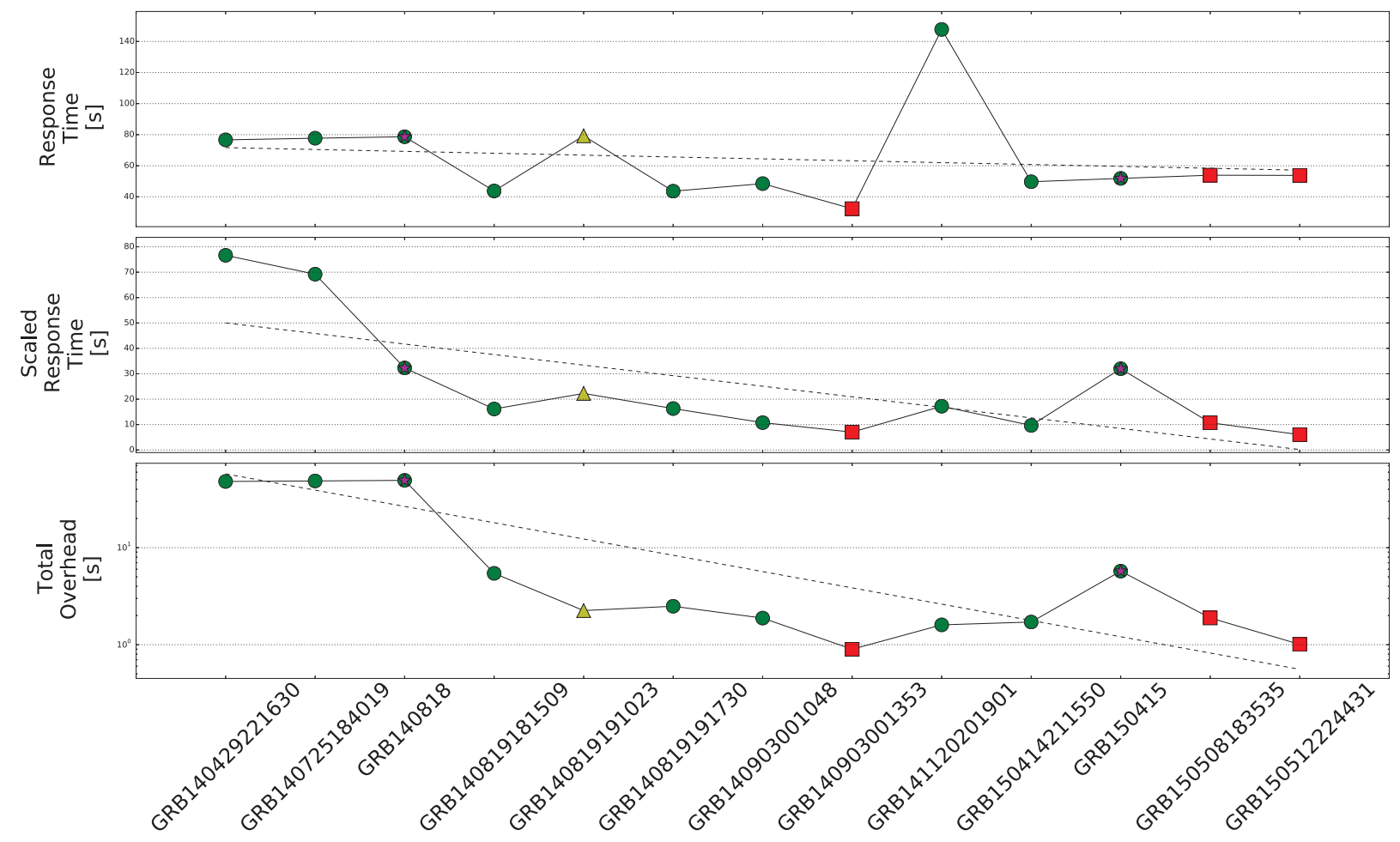

FIGURE 1. GRB observation total response time (top), response time scaled by the angular distance of the repointing (middle) and the data acquisition system time overhead (bottom) for several real and fake GRB observations. Figure reproduced from [10].

$\mathrm{GeV})$. This reduction in threshold, combined with the still large effective area $\left(>10^{4} \mathrm{~m}^{2}\right)$ has substantially improved the changes of a GRB detection. The combination of H.E.S.S. II with facilities at other wavelengths would allow the study of GRBs in ways never before possible.

\section{The H.E.S.S. II Rapid Repointing System}

As the fall of in intensity of GRBs after the initial burst is quite rapid, in order to maximise the chance of a significant detection wth the H.E.S.S. array, we must ensure the telescopes are able to slew to the target positions and begin observations as soon as possible after the GRB. This repointing time is minimised firstly by the significant upgrade in drive system performance of CT 5 over the original H.E.S.S. telescope (see [8] for details),allowing the instrument to perform a $180^{\circ}$ rotation in only 110 seconds. In addition the CT 5 is also able to point in reverse mode, enabling the telescope to slew through zenith allowing it to arrive on target much faster for GRBs that would otherwise require a long slew in azimuth.

In order to ensure a response to GRB alerts as soon as possible a fully automatic array repointing system has been implemented when a GRB alert matching the observation criteria is received. This system begins observations immediately after an alert is received, initiating a special purpose run type, which minimises the hardware and software state transitions to ensure the fastest possible repointings (see [9]). This system is regularly checked by the injection of fake GRBs into the system. Figure 1 shows the responce time of the repointing system to both real and fake bursts over time, demonstrating the optimisation of system responce time. 


\section{GRB Observations with H.E.S.S. II}

\section{The GCN System}

H.E.S.S. currently receives GRB alerts and localisations through the Gamma-ray Coordinates Network (GCN) [11], which automatically distributes the localisation of GRB detections from a number of instruments in different energy ranges typically in less than several minutes from burst detection. H.E.S.S. reacts to GRB alerts from two instruments, firstly the Fermi Gamma-ray Burst Monitor (GBM) [12] provides around 200 GRB localisations a year with an accuracy of 2-5 degrees [13]. In addition H.E.S.S. also reacts to alerts from the Swift Burst Alert Telescope (BAT) [14], which issues around 90 alerts per year with arc-minute level localisation accuracy.

\section{Selection Criteria}

GRB follow-ups are selected by H.E.S.S. using cuts based on the observability of the GRB position from the H.E.S.S. site and the likely presence of high energy emission. H.E.S.S. GRB observations are split into two categories, prompt and afterglow observations.

Prompt GRBs are classified as those where the burst occurs during H.E.S.S observation time and an array repointing can begin immediately. For prompt GRBs only observability criteria are applied, only requiring the GRB position to be visible for 30 minutes or more above 60 degrees zenith angle. Observations then proceed for 2 hours or until the source falls below 60 degrees zenith angle. If further (more accurate) GRB positions are issued by any instrument the H.E.S.S. observation position can then be updated to observe this point.

Afterglow observations are defined as those where the GRB position is not immediately observable from the H.E.S.S. site. These bursts are subject to cuts on both the observability as well as the likelihood of high energy emission. Stronger observability cuts are applied to such bursts, requiring the GRB to observable for at least 30 minutes above 45 degrees zenith angle. Additionally cuts are placed on the redshift of the GRB where available. Most often no redshift is available, for such bursts a maximum of 4 hours observation delay is accepted, increasing to 6 hours for redshift below 1, 12 hours for redshift below 0.3 and 24 hours for redshifts below 0.1 . For such afterglow observations a human is included in the decision making process, allowing exceptions to be made for bursts of particular interests, for example if a confirmed high energy Fermi-LAT detection has been made.

\section{GRB 140818B}

GRB 140818B was a long GRB detected by a number of dedicated GRB instruments (Fermi-GBM, Swift-BAT \& XRT) at 18:44:16 UTC. This event took place within the Fermi-LAT field of view, however no detection was made by this instrument. A GRB alert was issued through the GCN system and the alert reached the H.E.S.S. observatory at 18:45, immediately triggering an automated array repointing. Observations began at 18:46, with a delay of 117 seconds after burst detection and only 78 seconds after the alert was received.In total H.E.S.S. took 4 consecutive data taking runs with CTs 1-5, with a total of almost 2 hours observation time.

Analysis of this source position was then made using low energy optimised mono event analysis (CT5 only) [15]. Figure 2 shows a significance map of the region surrounding the Swift-BAT reconstructed GRB position, with no significant emission being found. Integral (table 1) and differential (figure 3) upper limits were therefore extracted from the GRB position.

TABLE 1. Integral flux upper limits (99\% confidence level above $100 \mathrm{GeV}$ ) at the Swift-BAT reconstructed position for the 4 H.E.S.S. data taking runs (assuming a photon index of 2)

\begin{tabular}{ccc}
\hline Run & Time after burst $(\mathrm{s})$ & Integral Flux Limit $\left(\mathrm{cm}^{-2} \mathrm{~s}^{-1}\right)$ \\
\hline 1 & $117-1758$ & $3.9 \times 10^{-11}$ \\
2 & $1865-3544$ & $2.6 \times 10^{-11}$ \\
3 & $3613-5298$ & $5.1 \times 10^{-11}$ \\
4 & $5365-7023$ & $1.8 \times 10^{-11}$
\end{tabular}




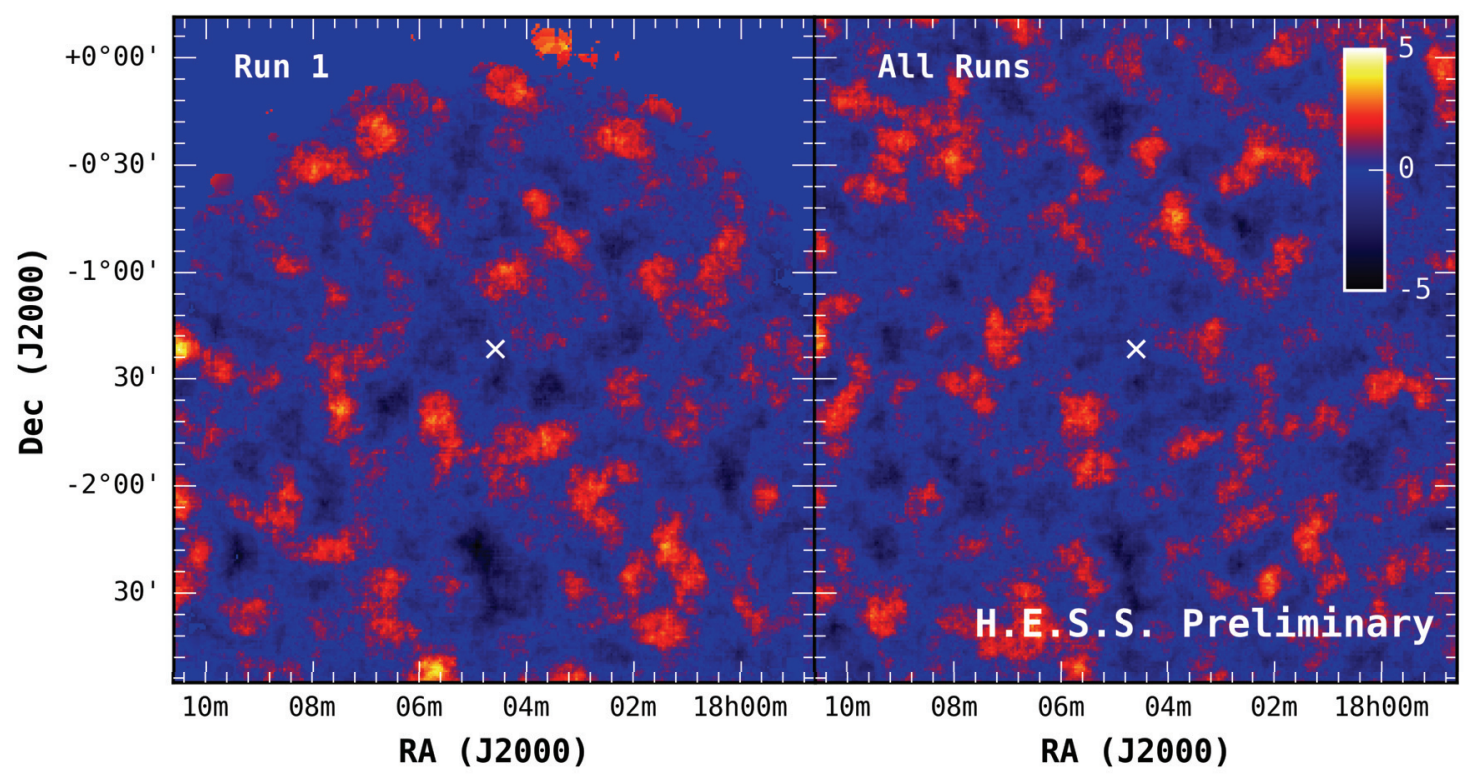

FIGURE 2. Significance map of the region surrounding GRB 140818B for the first observation run made by H.E.S.S. and the sum of all 4 runs (white cross marks Swift-BAT reconstructed position). Data was analysed using mono event analysis.

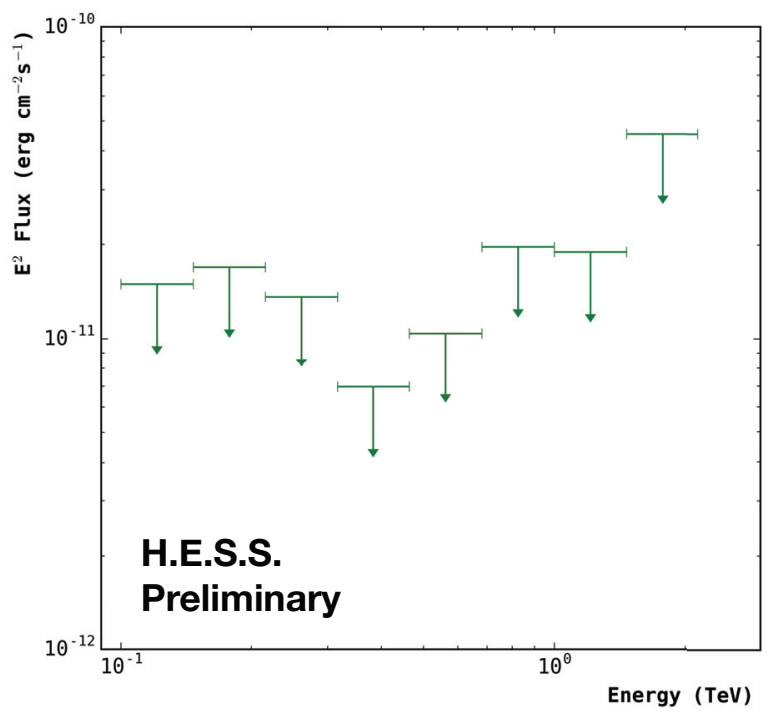

FIGURE 3. Differential flux upper limits (99\% confidence) for the first observation run made by H.E.S.S.. 


\section{Conclusions}

Taking into account the delay of the GCN system, the hardware and software overheads, and the repointing time of the array we expect to arrive at the GRB target position within 2-3 minutes for many possible observation positions. Analysis of simulated data shows that we expect to be sensitive down to around $50 \mathrm{GeV}$, greatly overlapping with the observed range of energies already seen by Fermi-LAT, with a greatly increased effective area. The first mono analysis of H.E.S.S. II data taken on GRB 140818B reveals no significant emission. However, this shows a stable and sensitive low energy analysis and providing upper limits on emission down to $100 \mathrm{GeV}$.

\section{Acknowledgements}

The support of the Namibian authorities and of the University of Namibia in facilitating the construction and operation of H.E.S.S. is gratefully acknowledged, as is the support by the German Ministry for Education and Research (BMBF), the Max Planck Society, the German Research Foundation (DFG), the French Ministry for Research, the CNRSIN2P3 and the Astroparticle Interdisciplinary Programme of the CNRS, the U.K. Science and Technology Facilities Council (STFC), the IPNP of the Charles University, the Czech Science Foundation, the Polish Ministry of Science and Higher Education, the South African Department of Science and Technology and National Research Foundation, the University of Namibia, the Innsbruck University, the Austrian Science Fund (FWF), and the Austrian Federal Ministry for Science, Research and Economy, and by the University of Adelaide and the Australian Research Council. We appreciate the excellent work of the technical support staff in Berlin, Durham, Hamburg, Heidelberg, Palaiseau, Paris, Saclay, and in Namibia in the construction and operation of the equipment. This work benefited from services provided by the H.E.S.S. Virtual Organisation, supported by the national resource providers of the EGI Federation.

\section{REFERENCES}

[1] M. Ackermann, M. Ajello, K. Asano. et al The First Fermi-LAT Gamma-Ray Burst Catalog. The Astrophysical Journal Supplement, Volume 209, 1,90, 2013.

[2] F. Aharonian, A.G. Akhperjanian, U. Barres de Almeida, et al. HESS observations of $\gamma$-ray bursts in 20032007. Astronomy and Astrophysics, Volume 495, 2, 505, 2009.

[3] J. Albert, E. Aliu, H. Anderhub, et al. MAGIC upper limits on the very high energy emission from GRBs. The Astrophysical Journal, Volume 667, 1, 358, 2007.

[4] E. Aliu, T. Aune, A. Barnacka, et al. Constraints on Very High Energy Emission from GRB 130427A. The Astrophysical Journal Letters, Volume 795, 1, 2014.

[5] A. U. Abeysekara, R. Alfaro, C. Alvarez, et al. Search for gamma-rays from the unusually bright GRB 130427A with the HAWC Gamma-ray Observatory. The Astrophysical Journal, Volume 800, 2, 78, 6, 2015.

[6] F. Halzen. Pionic photons and neutrinos from cosmic ray accelerators. Astroparticle Physics, 43, $155,2013$.

[7] A.A. Abdo, M. Ackermann, M. Ajello, et al. A limit on the variation of the speed of light arising from quantum gravity effects. Nature, 462, 7271, 331

[8] P. Hofverberg, R. Kankanyan, M. Panter, et al. Commissioning and initial performance of the H.E.S.S. II drive system. Proceedings of the 33rd International Cosmic Ray Conference, 2013.

[9] A. Balzer, M. Füssling, P. Hofverberg et al. The Performance of the H.E.S.S. Target of Opportunity Alert System. Proceedings of CHEP 2015.

[10] R.D. Parsons, A. Balzer, M. Füssling et al. The H.E.S.S. II GRB Program. Proceedings of the 34th International Cosmic Ray Conference, 2015

[11] The Gamma-ray Coordinates Network http://gcn.gscfc.nasa.gov

[12] C. Meegan, G. Lichti, P. N. Bhat, et al. The Fermi Gamma-Ray Burst Monitor. The Astrophysical Journal, Volume 702, 1, 791-804, 2009

[13] V. Connaughton, M. S. Briggs, A. Goldstein, et al. Localization of Gamma-Ray Bursts using the Fermi Gamma-Ray Burst Monitor. The Astrophysical Journal Supplement, Volume 216, 2, 27, 2015.

[14] S. D. Barthelmy, L. M. Barbier, J. R. Cummings et al. The Burst Alert Telescope (BAT) on the SWIFT Midex Mission. Space Science Reviews, 120, 143-164, 2005

[15] R.D. Parsons, M. Gajdus, and T. Murach. H.E.S.S. II Data analysis with ImPACT. Proceedings of the 34th International Cosmic Ray Conference, 2015 